\section{Transmitter Noise Considerations in Super-Nyquist FMCW Radar Design}

\section{Ash and P. V. Brennan}

In this letter, we introduce a design consideration for super-Nyquis FMCW radar systems relating to transmitter to receiver noise leakage. Phase noise curves comparing the fundamental DDS frequency and its $2^{\text {nd }}$ super-Nyquist image show a significant increase in relative noise level at all offsets from the carrier. This results in a reduced transmitter signal-to-noise ratio. It is shown with a simple example that this can lead to a significant degradation in receiver sensitivity if not managed properly. We provide a design rule-of-thumb that can be used by radar designers to avoid, or mitigate, this problem.

Introduction: Frequency modulated continuous wave (FMCW) radar is ubiquitous due to its simplistic, and relatively low-cost design. The emergence of digital devices for the generation of frequency modulated signals, such as direct digital synthesisers (DDS), poses a new set of design considerations for these systems. DDS-based FMCW radar has been used in imaging radar [1], automotive radar [2], and weather radar [3]. More recently, the use of the super-Nyquist technique has been explored to save a down-conversion process in receivers, which theoretically can improve sensitivity [4]. For long range FMCW radar applications, and the measurement of weak targets and short range, it is paramount to design with sensitivity in mind.

FMCW radar, by design, requires isolation of transmission and reception channels, normally with the use of separate antennas for each [5]. This demands an analysis into how much coupling the receiver can afford in order to avoid its saturation by the continuously transmitted radar signal [6]. The appropriate isolation between the transmitter and receiver is usually achieved with a suitable spatial separation of the antennas. The introduction of the super-Nyquist technique adds another factor to consider, that is the in-band noise associated with the DDS.

A DDS is a digital device whose fundamental output frequency range is defined by the frequency at which its digital circuitry is clocked, specifically its maximum theoretical output frequency is half of its digital clock frequency. However, the output spectrum of the DDS is not purely composed of the fundamental frequency. As it is a digital device, employing a zero order hold DAC, its output spectrum can be modelled a convolution of the spectrum of the fundamental frequency and the spectrum of a square pulse of the width of one cycle of the digital clock, which results in the well-known sinc function whose first-null appears at the digital clock frequency. Hence, the fundamental DDS signal aliases around half the digital clock frequency to form multiple images in what are known as Nyquist zones [7]. These images can be isolated with filtering allowing for the use of DDS devices as signal generators of frequencies beyond the specified fundamental output bandwidth.

In this letter, we show that the super-Nyquist technique must be used with caution when designing sensitive FMCW radar systems due to direct leakage of transmitter noise in to the receiver. We briefly summarise the super-Nyquist FMCW radar architecture, and discuss its advantages. We then provide a simple design rule-of-thumb, and show the effect of the increased noise on the radar receiver performance through an example. Finally, we make some suggestions to mitigate the effect of DDS noise when using the super-Nyquist technique.

Super-Nyquist FMCW Radar: FMCW radar systems can be designed to exploit the super-Nyquist technique to generate the FM radar signal directly at the required operating frequency using a DDS. Figure 1 shows a simple FMCW radar design based on this technique. The wanted radar signal in a particular Nyquist zone is isolated using a high-order bandpass filter. The signal is then amplified to an adequate power level, a portion of the signal is coupled with the receiver, and the remainder is passed through a power amplifier before being radiated with an antenna.

On reception, the echo signal is filtered and amplified before it is deramped by mixing with the coupled transmitter radar signal. The output of the mixer can then be further conditioned with amplification and filtering, before being digitised. The frequency content of the deramped signal can then be related to range by the following [8]:

$$
r=\frac{c f_{d}}{2 \alpha}
$$

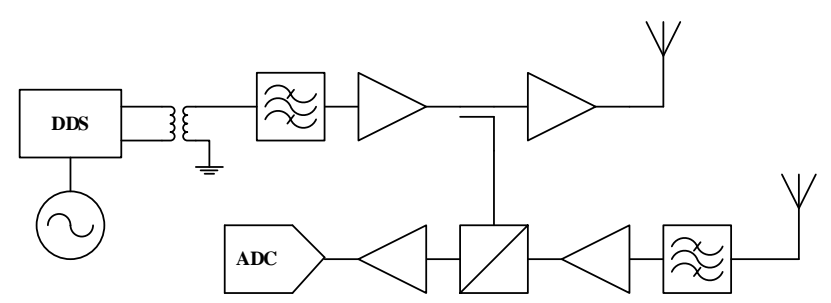

Fig. 1 Block diagram of a simple FMCW radar utilising the super-Nyquist technique. The wanted radar signal, located in a particular Nyquist zone, is isolated with a high-order band-pass filter before amplification and coupling with the receiver. On reception, only a signal mixer is required for deramping.

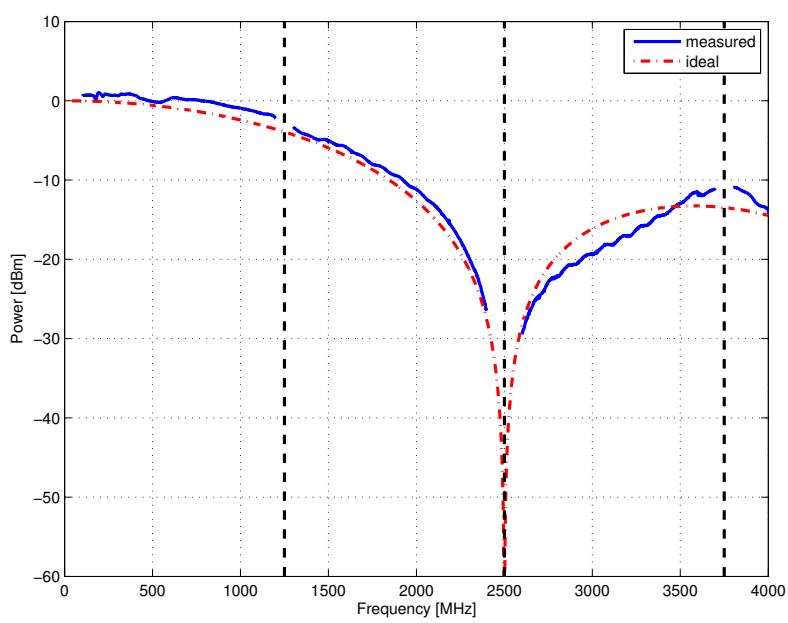

Fig. 2 Broadband AD9915 spectrum as measured using the AD9915 evaluation board. Cable losses, and losses associated with the TC1-1-13M+ balun used on the output of the DDS on the evaluation board, have been calibrated out of this measurement. The dashed lines indicate the location of Nyquist zones, associated with the digital clock frequency. The plot also includes an overlay of the ideal sinc spectrum, a result of the zero order hold architecture of the DDS output.

where $f_{d}$ is the deramped signal, and $\alpha$ is the chirp rate of the FM radar signal.

Using this technique, one can employ a homodyne receiver, saving a lossy mixing stage which would normally be required to translate the DDS fundamental to, and from, the operating frequency. Furthermore, it reduces the transmitter architecture complexity. Indeed, the job of the designer is simplified concerning dealing with spurious components generated by the extra mixing process.

Transmitter Noise Effects: In an FMCW radar system, the leakage of noise from the transmitter directly into the receiver can cause a reduction in sensitivity if not managed properly. It is in this respect where a superNyquist FMCW radar requires some consideration.

In this letter, we use at Analog Devices AD9915 DDS as an example to explore DDS performance beyond its fundamental frequency bandwidth. The AD9915 is a 2.5 GSPS, 12-bit DDS [9]. Measuring its output spectrum reveals the predicted sinc-shaped structure. There is a roll-off in signal level as more Nyquist zones are transitioned. This roll-off can be attributed to the sinc spectrum, and the specification of the output stage of the IC, which is likely to be optimised for the first Nyquist zone. Figure 2 shows the broadband spectrum of the AD9915 output as measured using the AD9915 evaluation board. Cable losses, and losses associated with the TC1-1-13M+ balun used on the output of the DDS on the evaluation board (based on the s-parameter files provided by the manufacturer [10]), have been calibrated out of this measurement. The calibration data for the balun is only available up to $4000 \mathrm{MHz}$, hence only the first three Nyquist zones have been fully plotted and will be considered in this letter.

The reduction in output power with increasing output frequency results in a degradation in output signal-to-noise ratio. This is normally not an issue when used in communications systems, where transmitter and receiver are significantly separated. However, as discussed, 


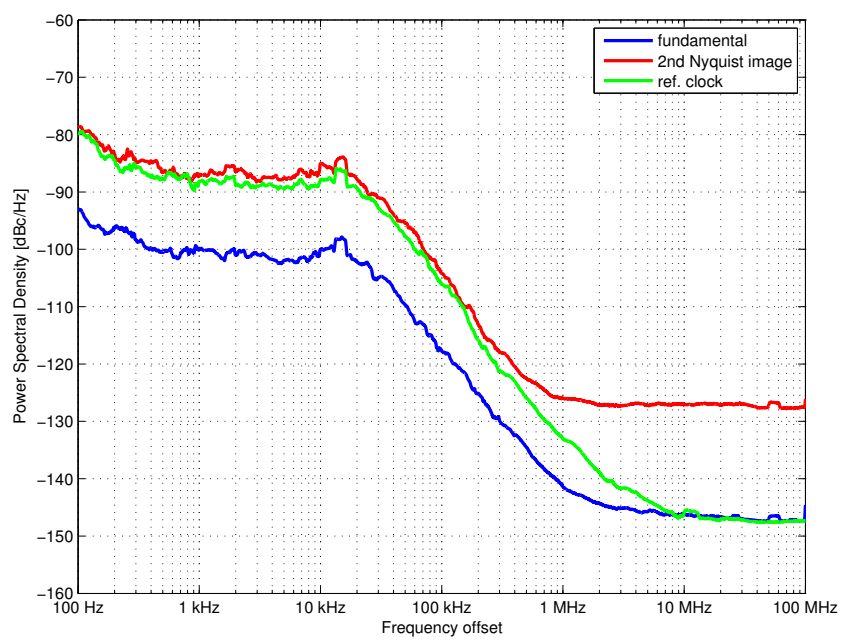

Fig. 3 Phase noise curves of a $600 \mathrm{MHz}$ fundamental signal, and its $2^{\text {nd }}$ Nyquist image at $3100 \mathrm{MHz}$, generated by an AD9915 DDS on the AD9915 evaluation board. The DDS is driven by an Analog Devices ADF4351 synthesiser tuned to $2500 \mathrm{MHz}$ whose phase noise curve is also included for reference.

transmitter to receiver coupling is a key issue in FMCW radar and hence the effective increase in transmitter noise level must be analysed.

Analysing the noise from the DDS that leaks from the transmitter, $P_{n, d}$, and the gain it experiences in the transmitter chain, a general ruleof-thumb for avoiding degradation of the receiver sensitivity is as follows:

$$
\begin{aligned}
& P_{n, t r}=\frac{P_{n, d} G_{t} F_{t}}{I_{t r}} \\
& P_{n, r}=k T_{o} B_{r} F_{r} \\
& P_{n, r}>P_{n, t r}
\end{aligned}
$$

where $P_{n, t r}$ is the transmitter noise at the receiver, $G_{t}$ is the gain of the transmitter, $F_{t}$ is the transmitter chain noise factor, $I_{t r}$ is the isolation between the transmitter and receiver, and $P_{n, r}$ is the receiver self-noise. In other words, the power of the noise from the transmitter as seen by the receiver must be less than the receiver self-noise power.

A plot comparing the phase noise curves of a $600 \mathrm{MHz}$ fundamental signal, and its $2^{\text {nd }}$ Nyquist image at $3100 \mathrm{MHz}$ (located in the $2^{\text {rd }}$ Nyquist zone), generated by an AD9915 DDS on the AD9915 evaluation board, is shown in Figure 3. The DDS is driven by an Analog Devices ADF4351 synthesiser tuned to $2500 \mathrm{MHz}$, whose phase noise curve is also included for reference. The output power of the fundamental is $+0.3 \mathrm{dBm}$ and the $2^{\text {nd }}$ Nyquist image is $-18.1 \mathrm{dBm}$.

If we consider the noise level at $1 \mathrm{MHz}$ offset from the carrier, the related noise level for the fundamental is c. $-142 \mathrm{dBc} / \mathrm{Hz}$, and $-125 \mathrm{dBc} / \mathrm{Hz}$ for the image. Factoring in the carrier signal level in each case, it can be seen that the phase noise power level stays almost the same in both cases, i.e. $P_{n, d} \approx-143 \mathrm{dBm} / \mathrm{Hz}$. Crucially though, the image signal will require a factor of 60 more gain than the fundamental to reach a particular signal level. This gain requirement increases with output frequency.

To see the effect of this noise level, we consider an example radar that requires a $1 \mathrm{~W}(+30 \mathrm{dBm})$ transmit power, with ideal amplifiers (i.e. $F_{t}=1$ ) and a good isolation of $I_{t r}=60 \mathrm{~dB}$ between the transmitter and receiver. The gain required to reach this power level is $+29.8 \mathrm{~dB}$ for the fundamental signal, and $+48.1 \mathrm{~dB}$ for the $2^{\text {nd }}$ Nyquist image signal. Entering these values into Equation (2):

$$
\begin{aligned}
P_{n, t r, \text { fund. }} & =-173.2 \mathrm{dBm} / \mathrm{Hz} \\
P_{n, t r, i m .} & =-154.9 \mathrm{dBm} / \mathrm{Hz}
\end{aligned}
$$

For a receiver with a $5 \mathrm{~dB}$ noise figure, hence $-169 \mathrm{dBm} / \mathrm{Hz}$ self-noise, Equation (4) is not satisfied. Indeed, the transmitter noise reduces the receiver sensitivity by as much as $14 \mathrm{~dB}$. Had the fundamental signal been used, the transmitter to receiver noise leakage would be less than the receiver self-noise, hence the receiver sensitivity would be unchanged.

The level of the effect sensitivity increases with Nyquist zone, and would be more marked if the noise generated in the transmitter chain following the DDS is considered. Hence, the designer of a super-Nyquist FMCW radar must be wary of this noise mechanism, and consider ways of mitigating its effect.

Mitigating the Effects of Transmitter Noise: Clearly, the use of the super-Nyquist effect can be detrimental to radar performance. However, considering Equation (2), the designer has control over the DDS noise, $P_{n, d}$, and the isolation between the transmitter in receiver, $I_{t r}$. Hence, its effect can be managed such that the transmitter noise falls below the receiver self-noise.

In order to decrease the DDS noise, the designer may need to go back to a traditional method of translating the fundamental DDS signal using a mixer, which is likely to be more noise efficient. In this case, it is the transmitter noise factor, $F_{t}$, that must be monitored. Using a low-noise amplifier directly after the DDS output normally keeps this low, even with a lossy mixer in the chain.

Alternatively, the designer can simply improve the isolation between the transmitter and receiver by increasing the spacing between the transmitter and receiver antennas until Equation (4) is met. This makes the super-Nyquist architecture ideal for multi-static radar, where the radar transmitters and receivers are not co-located. Hence, super-Nyquist FMCW radar would be an ideal solution for a low-cost, network of radar that can provide multi-perspective measurements of areas for applications such as perimeter security and monitoring.

Conclusion: In this letter, a design consideration for super-Nyquist FMCW radar systems is introduced. As a result of reduced signal-tonoise ratio associated with super-Nyquist transmitters, designers must be wary of reducing the sensitivity of the radar receiver. We have provided a design rule-of-thumb that can be used by radar designers to avoid, or mitigate, this problem. In addition, we have proposed that some situations may demand the use of translation of the fundamental DDS signal as a more noise efficient design, and suggested applications where the superNyquist technique makes for a good, low-cost solution.

Acknowledgment: The authors would like to thank EPSRC for funding the project that led to this discovery, grant ref. $\mathrm{EP} / \mathrm{K} 00767 \mathrm{X} / 1$.

M. Ash and P. V. Brennan (Department of Electronic and Electrical Engineering, University College London, London, UK)

E-mail:m.ash@ucl.ac.uk

\section{References}

1 Wang, W-Q., Peng, Q. and Cai, J.: 'Waveform-Diversity-Based Millimeter-Wave UAV SAR Remote Sensing', IEEE Transactions on Geoscience and Remote Sensing, 2009, 47, pp. 691-700

2 Stelzer, A., Kolmhofer, E. and Scheiblhofer, S.: 'Fast $77 \mathrm{GHz}$ chirps with direct digital synthesis and phase locked loop', Microwave Conference Proceedings, 2005. APMC 2005. Asia-Pacific Conference Proceedings, 2005

3 Gray, D.A., Viola, M., Moran, W., Samarasekera, S., May, P., Bates, B., Venkataraman, K., McCarroll, C., Ferguson, B. and McLaughlin, D.: 'WREN: A weather radar experimental network', International Conference on Electromagnetics in Advanced Applications (ICEAA), 2010, 2010

4 Huang, Y.: 'A Study of RF-Over-Fibre Based Active RFID Indoor Location System', Ph.D. thesis, University College London, UK, 2011

5 McGregor, J.A., Poulter, E.M. and Smith, M.J.: 'Switching system for single antenna operation of an S-band FMCW radar', IEE Proceedings - Radar, Sonar and Navigation, 1994, 141, pp. 241-248

$6 \mathrm{Li}, \mathrm{Z}$. and Wu K.: 'On the Leakage of FMCW Radar Front-End Receiver', Global Symposium on Millimeter Waves, 2008. GSMM 2008

7 Gentile, K.: 'Super-Nyquist Operation of the AD9912 Yields a High RF Output Signal', Analog Devices Application Note, AN-939, 2007

8 Stove, A.: 'Linear FMCW radar techniques', IEE Proceedings For Radar and Signal Processing, 1992, 5, pp. 343-350

9 Analog Devices: '2.5GSPS Direct Digital Synthesizer with 12-Bit DAC', AD9915 Datasheet, 2014, Rev. D

10 Mini-Circuits: 'TC1-1-13M+ RF Transformer S-Parameters', 2014, [Online]. Available: Mini-Circuits, http://www.minicircuits.com/. [Accessed November, 2014]. 